\title{
Fermi Surface Reconstruction and Electron Dynamics at the Charge-Density-Wave Transition in $\mathrm{TiSe}_{2}$
}

\author{
Patrick Knowles $\odot,{ }^{1}$ Bo Yang $\odot,{ }^{1}$ Takaki Muramatsu, ${ }^{1}$ Owen Moulding, ${ }^{1}$ Jonathan Buhot $\odot,{ }^{2}$ \\ Charles J. Sayers $\odot,^{3}$ Enrico Da Como ${ }^{3},{ }^{3}$ and Sven Friedemann $\oplus^{1, *}$ \\ ${ }^{1} \mathrm{HH}$ Wills Laboratory, University of Bristol, Bristol BS8 1TL, United Kingdom \\ ${ }^{2}$ High Field Magnet Laboratory, Radboud University, 6525 ED Nijmegen, The Netherlands \\ ${ }^{3}$ Centre for Nanoscience and Nanotechnology, Department of Physics, University of Bath, Bath BA2 7AY, United Kingdom
}

(Received 25 July 2019; accepted 20 March 2020; published 22 April 2020)

\begin{abstract}
The evolution of the charge carrier concentrations and mobilities are examined across the chargedensity-wave (CDW) transition in $\mathrm{TiSe}_{2}$. Combined quantum oscillation and magnetotransport measurements show that a small electron pocket dominates the electronic properties at low temperatures while an electron and hole pocket contribute at room temperature. At the CDW transition, an abrupt Fermi surface reconstruction and a minimum in the electron and hole mobilities are extracted from two-band and Kohler analysis of magnetotransport measurements. The minimum in the mobilities is associated with the overseen role of scattering from the softening $\mathrm{CDW}$ mode. With the carrier concentrations and dynamics dominated by the CDW and the associated bosonic mode, our results highlight $\mathrm{TiSe}_{2}$ as a prototypical system to study the Fermi surface reconstruction at a density-wave transition.
\end{abstract}

DOI: 10.1103/PhysRevLett.124.167602

The electronic properties of transition metal dichalcogenides (TMDs) are of fundamental and practical interest. Many TMDs can be tuned between semimetallic, semiconducting, and insulating behavior and thus allow us to access a plethora of different electronic characteristics. In addition, ordered states, e.g., due to charge-density-wave (CDW) formation $[1,2]$ or superconductivity [3,4] are present in many members of the family with open questions on the underlying mechanism. Many of these TMDs can be exfoliated to atomic monolayers providing new tuning parameters and novel physics through the reduced dimensionality [5-7].

$\mathrm{TiSe}_{2}$ is a prototypical material for strong electronic interactions driving the CDW formation via a condensation of excitons, i.e., pairs of electrons and holes [8,9]. Experimental and theoretical work have confirmed the relevance of the excitonic mechanism [10-12] that is widely accepted to work in cooperation with strong electron-phonon coupling [13-15].

Above the CDW transition temperature $T_{\mathrm{CDW}}=202 \mathrm{~K}$, $\mathrm{TiSe}_{2}$ is characterized by small carrier concentrations stemming from up to three selenium-derived hole bands with cylindrical topology at the $\Gamma$ point and a titaniumderived electron band with distorted and tilted ellipsoid topology present with threefold multiplicity at the $L$ point [16-20]. Whether these bands overlap in energy or have a band gap remains uncertain. Either way, the overlap or gap is small or comparable to thermal energies down to $50 \mathrm{~K}$.

Below the CDW transition temperature, the electronic structure of $\mathrm{TiSe}_{2}$ is dominated by a small electron pocket as shown by angle resolved photoemission spectroscopy
(ARPES) measurements [18]. Knowledge of how the electronic structure and electron dynamics evolve upon thermally melting the CDW in equilibrium, however, are outstanding. Several studies suggested that the Fermi surface reconstruction from the CDW order and scattering associated with the CDW mode has a negligible effect on the electronic structure and dynamics $[8,19,21,22]$. Rather a dominance of thermal occupation effects was suggested. In the past, studies of the charge carrier concentration were based on a single-band analysis of Hall effect and optical reflectivity measurements despite the evidence for two bands being present, while no measurements distinguishing the electron and hole dynamics across $T_{\mathrm{CDW}}$ have been reported $[23,24]$. Here, we use high-resolution magnetotransport and quantum oscillation (QO) measurements to extract the temperature dependence of the charge carrier concentration and mobility of the electron and hole band. We directly observe one quasi-ellipsoid Fermi surface at low temperatures which is identified as an electron pocket. This electron pocket and a hole pocket grow rapidly above $150 \mathrm{~K}$ showing evidence of an abrupt Fermi surface reconstruction and gapping of $75 \%$ of the charge carrier concentration in the CDW state. At the same time, we observe a minimum in the mobility on both the electron and hole pocket at $T_{\mathrm{CDW}}$, highlighting the importance of scattering from the CDW forming phononic and/or electronic modes.

Single crystals of $\mathrm{TiSe}_{2}$ were grown by chemical vapor transport at $580^{\circ} \mathrm{C}$ as detailed in Sec. SI of the Supplemental Material [25], and show a CDW transition at $T_{\mathrm{CDW}}=202 \mathrm{~K}$ consistent with other studies of highquality samples $[8,28-31]$. 

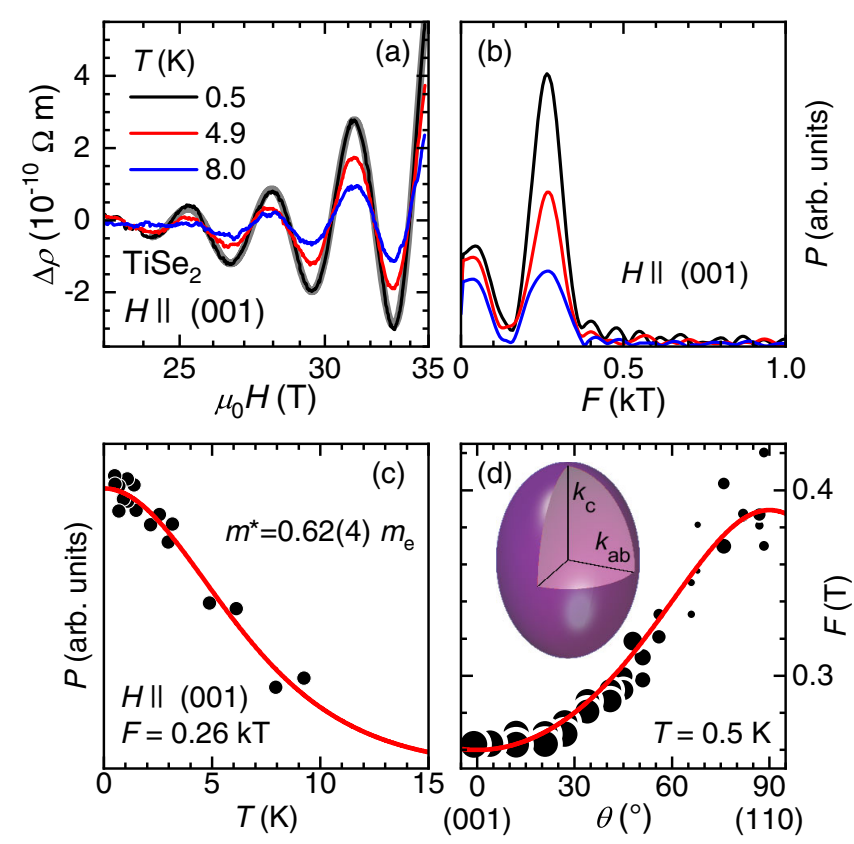

FIG. 1. Quantum oscillations in $\mathrm{TiSe}_{2}$. (a) The oscillatory component of the resistivity $\Delta \rho$ obtained after subtraction of a linear background. The thick gray line is a fit of a single frequency with Dingle damping factor and quadratic background correction to the data at $0.5 \mathrm{~K}$. (b) Fourier transform (FT) spectrum. (c) Temperature dependence of the FT amplitude of the $F=0.26 \mathrm{kT}$ oscillation. The red solid line is a Lifshitz-Kosevich fit to the data with an effective mass of $m_{e}^{\star}=0.62(4) m_{e}$, where $m_{e}$ denotes the bare electron mass. (d) Dependence of the oscillation frequency on zenith angle $\theta$ for fixed azimuthal angle $\phi=30^{\circ}$. Crystallographic directions are indicated on abscissa. Symbol size indicates oscillation amplitude. The solid red line represents a fit using an ellipsoid Fermi surface model as depicted in the inset and described in Sec. S III of the Supplemental Material [25].

The low-temperature electronic structure of our $\mathrm{TiSe}_{2}$ samples is dominated by an electron pocket as evident from the combination of $\mathrm{QO}$ and magnetotransport measurements. The QO data shown in Fig. 1 reveal a single frequency $F=0.26 \mathrm{kT}$ for magnetic fields parallel to the crystallographic $c$ direction, i.e., an orbit parallel to the basal plane. The increase of this frequency for orbits out of plane is well fitted by an ellipsoid shape [see Fig. 1(d) and Sec. S III of the Supplemental Material [25]]. This pocket is naturally associated with the $L$-point electron pocket observed by ARPES [18].

Magnetotransport measurements confirm the electrondominated character of our samples at low temperature as can be seen from the negative Hall resistivity $\rho_{x y}$ in Fig. 2(a). Consistent with earlier reports [8,32], $\rho_{x y}$ changes sign smoothly around $190 \mathrm{~K}$ (cf. Fig. S2 of the Supplemental Material [25]). The magnetoresistivity $\rho_{x x}(B)$ shown in Figs. 2(b)-2(e) is small and positive. We use a two-band model with one electron and one hole band to simultaneously fit $\rho_{x x}\left(\mu_{0} H\right)$ and $\rho_{x y}\left(\mu_{0} H\right)$ [25].
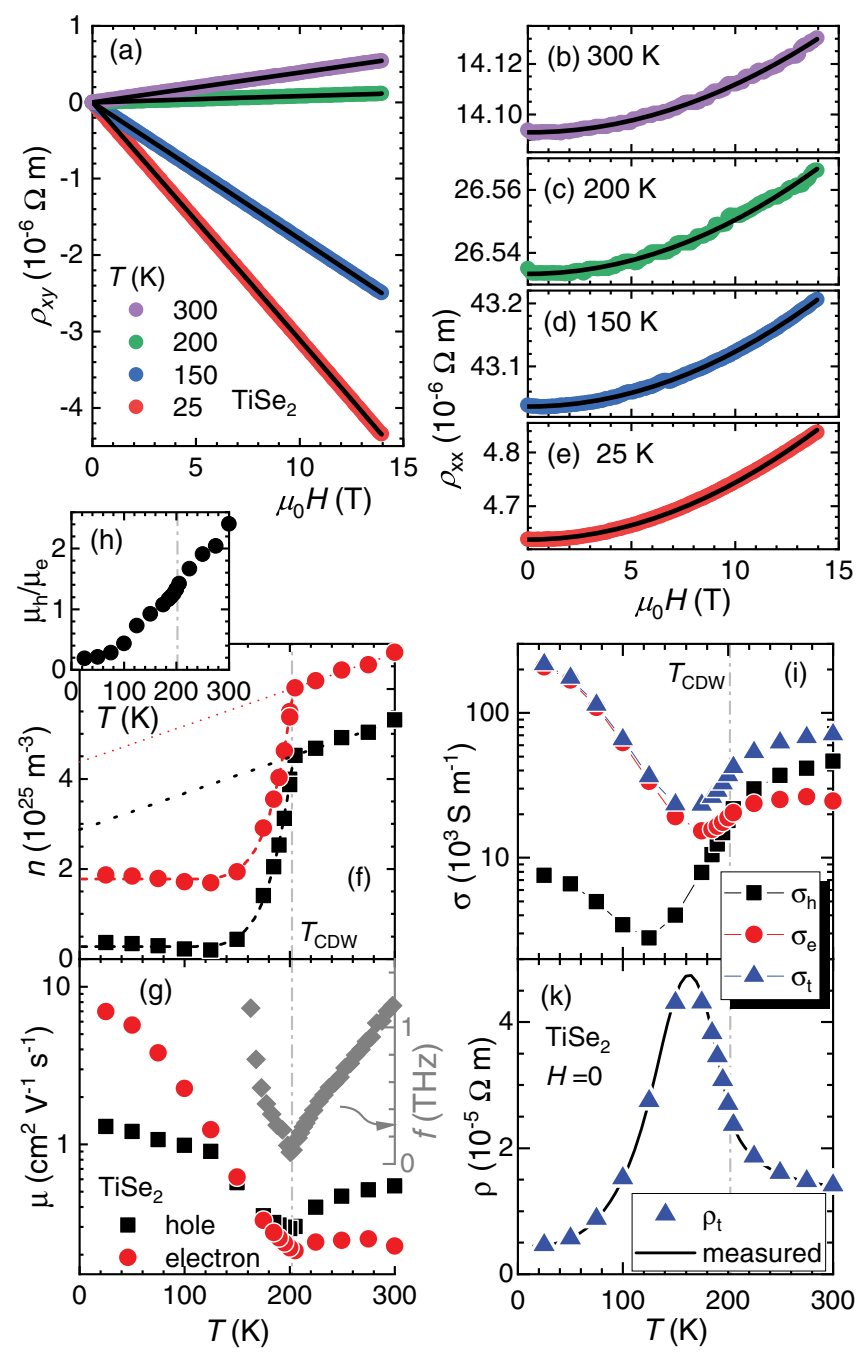

FIG. 2. Two-band analysis of magnetotransport in $\mathrm{TiSe}_{2}$. Transversal (a) and longitudinal (b)-(e) resistivities of $\mathrm{TiSe}_{2}$ at selected temperatures. Solid lines correspond to simultaneous two-band fits of both resistivities as described in Sec. S II of the Supplemental Material [25] giving charge carrier concentrations (f) and mobilities ( $\mathrm{g}$ ) of the hole and electron band. (g) includes frequency of $L$-mode phonon from Ref. [33] (adjusted for the reduced $T_{\mathrm{CDW}}$ of Ref. [33]). Dotted lines in (f) represent linear fits to $n_{e}(T)$ and $n_{h}(T)$ for $T \geq T_{\mathrm{CDW}}$. Dashed lines represent fits of the form $n_{e, h}(T)=n_{(e, h)}(T=0)+A \exp \left(-2 \Delta / k_{B} T\right)$. (h) Ratio of hole and electron mobility. Vertical dash-dotted line indicates $T_{\mathrm{CDW}}$. Standard errors as obtained from the nonlinear least-squares fits are smaller than symbol size. (i) Separate zerofield electron $\sigma_{e}$ and hole band conductivity $\sigma_{h}$ have been calculated from the mobilities and charge carrier concentrations shown in (f) and (g). Total conductivity is calculated as $\sigma_{t}=\sigma_{e}+\sigma_{h}$. Note the logarithmic scale. (k) Measured zerofield resistivity (solid line) compared to the calculated total resistivity $\rho_{t}=1 / \sigma_{t}$ (triangles).

The two-band fits shown as solid lines in Fig. 2 describe the data very well over the full temperature range.

The charge carrier concentrations $n_{e}, n_{h}$ and mobilities $\mu_{e}, \mu_{h}$ for the electron and hole band, respectively, are 
shown in Figs. 2(f) and 2(g). The low-temperature value of $n_{e}$ is in good agreement with our QO data and previous ARPES as well as heat capacity studies as summarized in Table S1 [25]. Thus the association of the observed QO frequency with the electron pocket is confirmed. The electron pocket observed in our QO measurements accounts for virtually the full low-temperature electronic heat capacity (cf. Table S1 [25]) highlighting the dominance of the electron pocket at low $T$. The hole concentration extracted within the two-band model is very small at lowest temperatures and is likely to correspond to impurity states as indicated by the low hole mobility. We note that a free-electron single-band model cannot describe the low-temperature magnetotransport. Most notably, the Hall coefficient $R_{H}=-3.1 \times 10^{-7} \mathrm{~m}^{3} \mathrm{C}^{-1}$ measured at lowest temperatures does not match with a free electron estimate for the electron pocket based on the QO results of $R_{H}^{\mathrm{QO}}=-1.75 \times 10^{-7} \mathrm{~m}^{3} \mathrm{C}^{-1}$.

At room temperature, the electron and hole concentrations $n_{e}$ and $n_{h}$ are comparable in magnitude [cf. Fig. 2(f)]. Above $T_{\mathrm{CDW}}, n_{e}$ and $n_{h}$ are associated with the 3D-like electron pocket at the $L$ point and the 2D-like hole pocket at the $\Gamma$ point as seen by ARPES studies $[17,18]$. The small linear temperature dependence of $n_{e}(T)$ and $n_{h}(T)$ above $210 \mathrm{~K}$ [dotted lines in Fig. 2(f)] is attributed to the varying thermal occupation of the two bands similar to the model of Watson et al. [21]. The slope of $n_{e}(T)$ and $n_{h}(T)$ for $T \geq T_{\mathrm{CDW}}$ suggests an effective mass for the electron band comparable to the free-electron mass. The linear behavior at high temperatures extrapolates to finite intercepts for both bands - these finite intercepts suggest a band overlap, i.e., not a gap, in the high-temperature phase above $T_{\mathrm{CDW}}$.

The charge carrier concentrations show a sharp drop below $T_{\mathrm{CDW}}$ and saturate below $150 \mathrm{~K}$. The drop is associated with condensation of electrons and holes into the CDW pair state and consequently with a Fermi surface reconstruction. The difference $\Delta n_{e, h}=n_{e, h}\left(T_{\mathrm{CDW}}\right)-$ $n_{e, h}(T=0)=4.2(2) \times 10^{25} \mathrm{~m}^{-3}$ marks the loss of charge carriers and thus the density of electron-hole pairs. For $T<T_{\mathrm{CDW}}, n_{e}(T)$ and $n_{h}(T)$ can be described by activated behavior as shown by dashed lines in Fig. 2(f). Fits of exponential form yield a gap $\Delta_{\mathrm{CDW}}=75(1) \mathrm{meV}$, an energy scale consistent with $T_{\mathrm{CDW}}=202 \mathrm{~K}$. The fact that the exponential form fits the data close to $T_{\mathrm{CDW}}$ suggests a finite gap up to $T_{\mathrm{CDW}}$. A finite gap has indeed been seen in ARPES studies [34-36] where the total gap $\Delta=\Delta_{\mathrm{CDW}}+$ $\Delta_{\text {off }}$ is a sum of a BCS-like temperature dependent gap $\Delta_{\mathrm{CDW}}$ with an onset at $T_{\mathrm{CDW}}$ on top of a weakly temperature dependent offset $\Delta_{\text {off }} \approx 70 \mathrm{meV}$. Our exponential fits are dominated by the temperature dependence of $n(T)$ just below $T_{\mathrm{CDW}}$, where $\Delta \approx \Delta_{\text {off }}$. The exponential form of $n_{e}(T)$ and $n_{h}(T)$ up to $T_{\mathrm{CDW}}$ and the good agreement of the gap value with $\Delta_{\text {off }}$ suggests a finite gap present above $T_{\mathrm{CDW}}$ potentially due to fluctuating electron-hole pairs that condense at $T_{\mathrm{CDW}}$ consistent with ARPES studies finding weak intensity from backfolded bands above $T_{\mathrm{CDW}}$ [11].

The Fermi-surface reconstruction is further supported by the Kohler analysis [37] presented in Fig. 3. The magnetoresistance follows a quadratic field dependence at $T \geq 50 \mathrm{~K}$. However, the quadratic coefficient (Kohler slope) $K$ shows a pronounced temperature dependence [cf. Fig. 3(b)]. Above $T_{\mathrm{CDW}}, K$ is virtually constant and accordingly curves of MR vs $\left(\mu_{0} H / \rho_{0}\right)^{2}$ collapse in Fig. 3(a). Below $T_{\mathrm{CDW}}$, however, $K$ rises very abruptly by more than an order of magnitude, passes a maximum at $150 \mathrm{~K}$ and saturates at a low-temperature value approximately double the room temperature value.

Kohler scaling and violations thereof have been observed in other CDW systems: In $\mathrm{VSe}_{2}$ and $\mathrm{NbSe}_{2}$, separate Kohler scaling is present below and above $T_{\mathrm{CDW}}$ with a small difference in slope at $T_{\mathrm{CDW}}[38,39]$. In $\mathrm{Ta}_{2} \mathrm{NiSe}_{7}$ and $\mathrm{NbSe}_{3}$, Kohler scaling is only obeyed above $T_{\mathrm{CDW}}$. In underdoped cuprate superconductors, Kohler scaling is observed at low temperature throughout $T_{\mathrm{CDW}}$ [40]. Our results on $\mathrm{TiSe}_{2}$ show a larger change in $K$ compared to
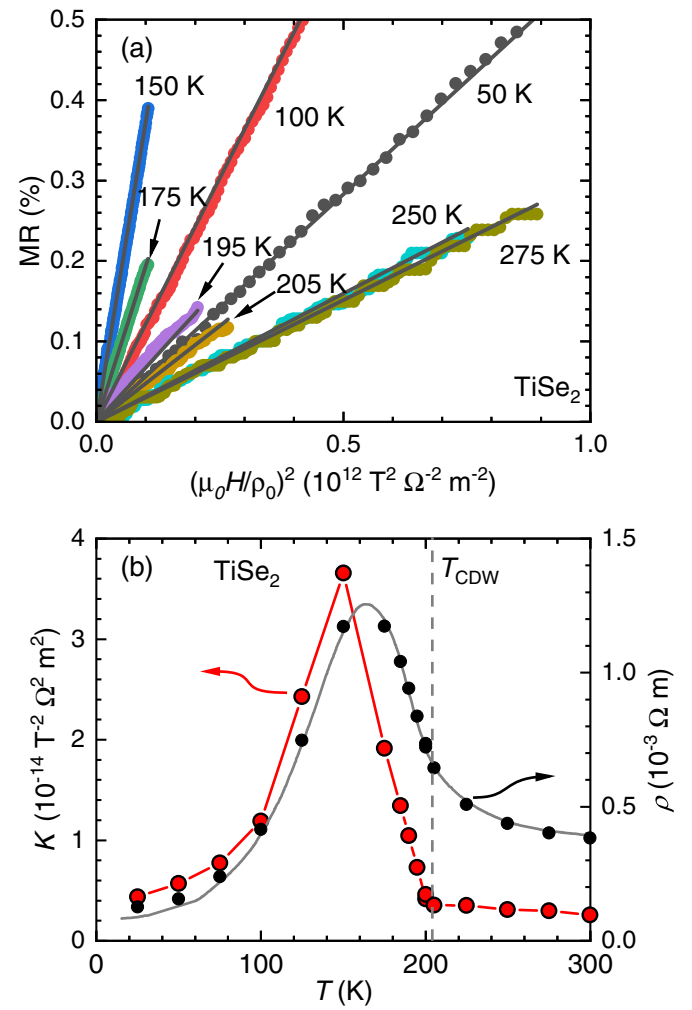

FIG. 3. Kohler analysis of the magnetoresistance in $\mathrm{TiSe}_{2}$. (a) Magnetoresistance $\mathrm{MR}=\left(\rho-\rho_{0}\right) / \rho_{0}$ plotted against $\left(\mu_{0} H / \rho_{0}\right)^{2}$ where $\rho_{0}=\rho(T, H=0)$ Solid lines are linear fits of $\mathrm{MR}=K\left(\mu_{0} H\right)^{2}$. (b) The Kohler slope $K(T)$ (left axis) is shown with the solid red line as a guide to the eye. The zero-field resistivity $\rho_{0}(T)$ (black circles, right axis) is shown together with $\rho(T)$ from Ref. [8] (solid line) where the latter has been scaled to our data. 
other compounds because a larger fraction of the Fermi surface is affected by the CDW.

Our data suggest the Fermi surface reconstruction is the main reason for the violation of Kohler scaling in $\mathrm{TiSe}_{2}$. The sharp rise and strong temperature dependence of $K$ below $T_{\mathrm{CDW}}$ can be due to (i) a reconstruction of the Fermi surface, (ii) an abrupt change in the anisotropy of the scattering time on the individual bands, or (iii) an abrupt change in the ratio of the scattering times of the electron and hole band, or a combination of the three. (iii) can be ruled out as this would manifest as an abrupt change of $\mu_{h} / \mu_{e}$ which is not observed [Fig. 2(h)]. (ii) May contribute to the change of $K$ but it is unlikely to be the primary cause. For the violation of Kohler's law to be dominated by changes to scattering time anisotropy, a drastic and abrupt change to the phonon spectrum would be required. This is unlikely to occur independent of the Fermi surface reconstruction. A moderate change of the phonon spectrum may occur as a consequence of the Fermi surface reconstruction through the electron-phonon coupling. Thus, the sudden change of $K$ at $T_{\mathrm{CDW}}$ is dominated by (i) a sudden reconstruction of the Fermi surface. This is in agreement with the sudden drop of the charge carrier concentrations [Fig. 2(f)].

The mobilities of the individual bands show very strong and nontrivial temperature dependencies (cf. Fig. 2(g)). At room temperature, the hole mobility is larger than the electron mobility while this is reversed at lowest temperatures. Both mobilities have a minimum at $T_{\mathrm{CDW}}$ naturally associated with strong scattering from the softening mode associated with the CDW formation. Indeed, the temperature dependence of the mobilities show a dip similar in shape to the energy dependence of the $L$-point phonon mode [33] [reproduced in Fig. 2(g)].

As noticed by Velebit et al. [23], the mobilities of the two bands are roughly equal at $T_{\mathrm{CDW}}$ as shown in Fig. 2(h). This equality highlights that scattering from the $L$-point mode is the dominant process as the phase space for scattering from the electron to the hole band involves the density of states in both bands and the electron-phonon coupling.

The temperature dependence of the separated electron $\left(\sigma_{e}\right)$ and hole $\left(\sigma_{h}\right)$ conductivity are presented in Fig. 2(i). They show a minimum around $\approx 170$ and $\approx 120 \mathrm{~K}$, respectively. The total conductivity $\sigma_{t}=\sigma_{e}+\sigma_{h}$ and the total resistivity $\rho_{t}=1 / \sigma_{t}$ are dominated by $\sigma_{e}$ and $\sigma_{h}$ in different temperature regimes. The comparison of the thus calculated $\rho_{t}$ and the measured $\rho_{x x}(H=0)$ in Fig. 2(k) highlights the accuracy of the parameters extracted from the two-band fits.

From the temperature dependencies of the individual and total conductivities we identify that (i) at high temperature holes dominate the total conductivity and (ii) the negative $d \rho / d T$ above $T_{\mathrm{CDW}}$ is a consequence of the hole mobility increasing with temperature above $T_{\mathrm{CDW}}$. (iii) The peak in
$\rho(T)$ is dominated by the loss of a large portion of charge carrier concentration of both holes and electrons due to the CDW with (iv) the positive $d \rho / d T$ at low temperatures arising due to the large increase in electron mobility towards lowest temperatures. (v) At low temperatures electrons dominate the conductivity. In summary, we conclude that the magnetotransport is dominated by the opening of the CDW gap and the scattering from the underlying bosonic mode.

Despite the smooth evolution of the resistivity, the magnetotransport behaviour provides direct evidence for an underlying abrupt Fermi surface reconstruction both as a large drop of the charge carrier concentrations and a sudden violation of Kohler's law below $T_{\mathrm{CDW}}$. The analysis shows the loss of both electrons and holes below $T_{\mathrm{CDW}}$ highlighting the strong coupling between them. The fact that a large fraction of the charge carrier concentration is involved in the formation of the CDW enables a clear view at the electronic scattering associated with the $L$-point $C D W$ mode. The minimum in the mobility at $T_{\mathrm{CDW}}$ is a direct match to the softening of the $L$-point mode and thus confirms that the dynamics of the charge carriers are directly linked to the dynamics of the CDW mode. Importantly, our measurements show that the strong scattering of the CDW mode causes the intriguing negative $d \rho / d T$ at room temperature. This makes $\mathrm{TiSe}_{2}$ uniquely suitable to observe the strong coupling of the CDW mode to the electronic states. The scattering of electrons from the softening CDW mode is obscured in other prototypical CDW systems like $\mathrm{NbSe}_{2}$ or $\mathrm{VSe}_{2}$ where only small portions of the Fermi surface are matched by the ordering wave vector and only these "hot" parts experience strong scattering [41,42]. Thus, $\mathrm{TiSe}_{2}$ is a prototypical system to study the effects of a Fermi surface reconstruction arising from a CDW. These results will be relevant to understand systems like cuprate and iron-pnictide superconductors [43,44].

Data are available at the University of Bristol data repository [45].

The authors thank Jasper van Wezel, Jans Henke, Antony Carrington, Martin Gradhand, Matthew Wattson, and Phil King for valuable discussion. The authors acknowledge support by the EPSRC under Grants No. EP/N026691/1, No. EP/L015544/1, and No. NS/ A000060/1, support of the HFML-RU/NWO, member of the European Magnetic Field Laboratory (EMFL), and funding from the European Research Council (ERC) under the European Union's Horizon 2020 research and innovation programme (Grant Agreement No. 715262-HPSuper).

*Sven.Friedemann@bristol.ac.uk

[1] J. A. Wilson, F. Di Salvo, and S. Mahajan, Charge-density waves and superlattices in the metallic layered transition metal dichalcogenides, Adv. Phys. 24, 117 (1975). 
[2] J. A. Wilson and A. Yoffe, The transition metal dichalcogenides discussion and interpretation of the observed optical, electrical and structural properties, Adv. Phys. 18, 193 (1969).

[3] E. Morosan, H. W. Zandbergen, B. S. Dennis, J. W. G. Bos, Y. Onose, T. Klimczuk, A. P. Ramirez, N. P. Ong, and R. J. Cava, Superconductivity in $\mathrm{Cu}_{x} \mathrm{TiSe}_{2}$, Nat. Phys. 2, 544 (2006).

[4] A. F. Kusmartseva, B. Sipos, H. Berger, L. Forró, and E. Tutiš, Pressure Induced Superconductivity in Pristine $1 T-\mathrm{TiSe}_{2}$, Phys. Rev. Lett. 103, 236401 (2009).

[5] X. Xi, L. Zhao, Z. Wang, H. Berger, L. Forró, J. Shan, and K. F. Mak, Strongly enhanced charge-density-wave order in monolayer $\mathrm{NbSe}_{2}$, Nat. Nanotechnol. 10, 765 (2015).

[6] X. Xi, Z. Wang, W. Zhao, J.-H. Park, K. T. Law, H. Berger, L. Forró, J. Shan, and K. F. Mak, Ising pairing in superconducting $\mathrm{NbSe}_{2}$ atomic layers, Nat. Phys. 12, 139 (2016).

[7] B. Singh, C.-H. Hsu, W.-F. Tsai, V. M. Pereira, and H. Lin, Stable charge density wave phase in a $1 T-\mathrm{TiSe}_{2}$ monolayer, Phys. Rev. B 95, 245136 (2017).

[8] F. Di Salvo, D. Moncton, and J. Waszczak, Electronic properties and superlattice formation in the semimetal TiSe $_{2}$, Phys. Rev. B 14, 4321 (1976).

[9] J. A. Wilson, Modelling the contrasting semimetallic characters of $\mathrm{TiS}_{2}$ and $\mathrm{TiSe}_{2}$, Phys. Status Solidi 86, 11 (1978).

[10] S. Hellmann, T. Rohwer, M. Kalläne, K. Hanff, C. Sohrt, A. Stange, A. Carr, M. M. Murnane, H. C. Kapteyn, L. Kipp et al., Time-domain classification of charge-density-wave insulators, Nat. Commun. 3, 1069 (2012).

[11] H. Cercellier, C. Monney, F. Clerc, C. Battaglia, L. Despont, M. G. Garnier, H. Beck, P. Aebi, L. Patthey, H. Berger et al., Evidence for an Excitonic Insulator Phase in $1 T-\mathrm{TiSe}_{2}$, Phys. Rev. Lett. 99, 146403 (2007).

[12] A. Kogar, M. S. Rak, S. Vig, A. A. Husain, F. Flicker, Y. I. Joe, L. Venema, G. J. MacDougall, T. C. Chiang, E. Fradkin et al., Signatures of exciton condensation in a transition metal dichalcogenide, Science 358, 1314 (2017).

[13] H. Hedayat, C. J. Sayers, D. Bugini, C. Dallera, D. Wolverson, T. Batten, S. Karbassi, S. Friedemann, G. Cerullo, J. van Wezel et al., Excitonic and lattice contributions to the charge density wave in $1 T-\mathrm{TiSe}_{2}$ revealed by a phonon bottleneck, Phys. Rev. Research 1, 023029 (2019).

[14] M. Porer, U. Leierseder, J.-M. Ménard, H. Dachraoui, L. Mouchliadis, I. E. Perakis, U. Heinzmann, J. Demsar, K. Rossnagel, and R. Huber, Non-thermal separation of electronic and structural orders in a persisting charge density wave, Nat. Mater. 13, 857 (2014).

[15] J. van Wezel, P. Nahai-Williamson, and S. S. Saxena, Exciton-phonon-driven charge density wave in $\mathrm{TiSe}_{2}$, Phys. Rev. B 81, 165109 (2010).

[16] R. Bianco, M. Calandra, and F. Mauri, Electronic and vibrational properties of $\mathrm{TiSe}_{2}$ in the charge-density-wave phase from first principles, Phys. Rev. B 92, 094107 (2015).

[17] J. C. E. Rasch, T. Stemmler, B. Müller, L. Dudy, and R. Manzke, $1 T-\mathrm{TiSe}_{2}$ : Semimetal or Semiconductor? Phys. Rev. Lett. 101, 237602 (2008).

[18] M. D. Watson, O. J. Clark, F. Mazzola, I. Marković, V. Sunko, T. K. Kim, K. Rossnagel, and P. D. C. King,
Orbital- and $k_{z}$-Selective Hybridization of Se $4 p$ and Ti $3 d$ States in the Charge Density Wave Phase of TiSe ${ }_{2}$, Phys. Rev. Lett. 122, 076404 (2019).

[19] T. Pillo, J. Hayoz, H. Berger, F. Lévy, L. Schlapbach, and P. Aebi, Photoemission of bands above the Fermi level: The excitonic insulator phase transition in $1 T-\mathrm{TiSe}_{2}$, Phys. Rev. B 61, 16213 (2000).

[20] We use the high-temperature notation of the Brillouin zone throughout the Letter. In the low-temperature phase the high-temperature $L$ point folds back onto the hightemperature $\Gamma$ point.

[21] M. D. Watson, A. M. Beales, and P. D. C. King, On the origin of the anomalous peak in the resistivity of $\mathrm{TiSe}_{2}$, Phys. Rev. B 99, 195142 (2019).

[22] C. Monney, E. F. Schwier, M. G. Garnier, N. Mariotti, C. Didiot, H. Cercellier, J. Marcus, H. Berger, A. N. Titov, H. Beck et al., Probing the exciton condensate phase in $1 T-\mathrm{TiSe}_{2}$ with photoemission, New J. Phys. 12, 125019 (2010).

[23] K. Velebit, P. Popčević, I. Batistić, M. Eichler, H. Berger, L. Forró, M. Dressel, N. Barišić, and E. Tutiš, Scatteringdominated high-temperature phase of $1 T-\mathrm{TiSe}_{2}: \mathrm{An}$ optical conductivity study, Phys. Rev. B 94, 075105 (2016).

[24] G. Li, W. Z. Hu, D. Qian, D. Hsieh, M. Z. Hasan, E. Morosan, R. J. Cava, and N. L. Wang, Semimetal-to-Semimetal Charge Density Wave Transition in $1 T-\mathrm{TiSe}_{2}$, Phys. Rev. Lett. 99, 027404 (2007).

[25] See Supplemental Material at http://link.aps.org/ supplemental/10.1103/PhysRevLett.124.167602 for details of the two-band analysis on $\mathrm{TiSe}_{2}$, which includes Refs. [26] and details of the Fermi-surface analysis and comparison to complementary work, which includes Refs. [27].

[26] K. K. Huynh, Y. Tanabe, T. Urata, S. Heguri, K. Tanigaki, T. Kida, and M. Hagiwara, Mobility spectrum analytical approach for intrinsic band picture of $\mathrm{Ba}(\mathrm{FeAs})_{2}$, New J. Phys. 16, 093062 (2014).

[27] R. A. Craven, F. J. Di Salvo, and F. S. L. Hsu, Mechanisms for the $200 \mathrm{~K}$ transition in $\mathrm{TiSe}_{2}$ : A measurement of the specific heat Author links open overlay panel, Solid State Commun. 25, 39 (1978).

[28] I. Taguchi, M. Asai, Y. Watanabe, and M. Oka, Transport properties of iodine-free $\mathrm{TiSe}_{2}$, Physica (Amsterdam) 105(B +C), 146 (1981).

[29] S. H. Huang, G. J. Shu, W. W. Pai, H. L. Liu, and F. C. Chou, Tunable Se vacancy defects and the unconventional charge density wave in $1 T-\mathrm{TiSe}_{2-\delta}$, Phys. Rev. B 95, 045310 (2017).

[30] J. M. Moya, C.-L. Huang, J. Choe, G. Costin, M. S. Foster, and E. Morosan, Effect of synthesis conditions on the electrical resistivity of $\mathrm{TiSe}_{2}$, Phys. Rev. Mater. 3, 084005 (2019).

[31] B. Hildebrand, C. Didiot, A. M. Novello, G. Monney, A. Scarfato, A. Ubaldini, H. Berger, D. R. Bowler, C. Renner, and P. Aebi, Doping Nature of Native Defects in $1 T-\mathrm{TiSe}_{2}$, Phys. Rev. Lett. 112, 197001 (2014).

[32] D. J. Campbell, C. Eckberg, P. Y. Zavalij, H.-H. Kung, E. Razzoli, M. Michiardi, C. Jozwiak, A. Bostwick, E. Rotenberg, A. Damascelli et al., Intrinsic insulating ground state in transition metal dichalcogenide $\mathrm{TiSe}_{2}$, Phys. Rev. Mater. 3, 053402 (2019). 
[33] M. Holt, P. Zschack, H. Hong, M. Y. Chou, and T.-C. Chiang, X-Ray Studies of Phonon Softening in TiSe 2 , Phys. Rev. Lett. 86, 3799 (2001).

[34] P. Chen, Y.-H. Chan, X.-Y. Fang, S.-K. Mo, Z. Hussain, A.-V. Fedorov, M. Y. Chou, and T.-C. Chiang, Hidden order and dimensional crossover of the charge density waves in $\mathrm{TiSe}_{2}$, Sci. Rep. 6, 37910 (2016).

[35] M.-L. Mottas, T. Jaouen, B. Hildebrand, M. Rumo, F. Vanini, E. Razzoli, E. Giannini, C. Barreteau, D. R. Bowler, C. Monney et al., Semimetal-to-semiconductor transition and charge-density-wave suppression in $1 T-\mathrm{TiSe}_{2-x} S_{x}$ single crystals, Phys. Rev. B 99, 155103 (2019).

[36] C. Monney, E. F. Schwier, M. G. Garnier, N. Mariotti, C. Didiot, H. Beck, P. Aebi, H. Cercellier, J. Marcus, C. Battaglia et al., Temperature-dependent photoemission on $1 T-\mathrm{TiSe}_{2}$ : Interpretation within the exciton condensate phase model, Phys. Rev. B 81, 155104 (2010).

[37] B. Pippard, Magnetoresistance in Metals (Cambridge University Press, Cambridge, England, 1989).

[38] Y. Xue, Y. Zhang, H. Wang, S. Lin, Y. Li, J.-Y. Dai, and S. P. Lau, Thickness-dependent magnetotransport properties in $1 \mathrm{~T} \mathrm{VSe}_{2}$ single crystals prepared by chemical vapor deposition, Nanotechnology 31, 145712 (2020).

[39] K. Noto, S. Morohashi, K. Arikawa, and Y. Muto, Temperature and magnetic field dependence of the electrical resistance in pure and Fe-doped $2 \mathrm{H}-\mathrm{NbSe}_{2}$, Physica (Amsterdam) 99(B+C), 204 (1980).
[40] M. K. Chan, M. J. Veit, C. J. Dorow, Y. Ge, Y. Li, W. Tabis, Y. Tang, X. Zhao, N. Barišić, and M. Greven, In-Plane Magnetoresistance Obeys Kohler's Rule in the Pseudogap Phase of Cuprate Superconductors, Phys. Rev. Lett. 113, 177005 (2014).

[41] K. Rossnagel, O. Seifarth, L. Kipp, M. Skibowski, D. Voß, P. Krüger, A. Mazur, and J. Pollmann, Fermi surface of $2 \mathrm{H}-\mathrm{NbSe}_{2}$ and its implications on the charge-densitywave mechanism, Phys. Rev. B 64, 235119 (2001).

[42] V. N. Strocov, M. Shi, M. Kobayashi, C. Monney, X. Wang, J. Krempasky, T. Schmitt, L. Patthey, H. Berger, and P. Blaha, Three-Dimensional Electron Realm in $\mathrm{VSe}_{2}$ by Soft-X-Ray Photoelectron Spectroscopy: Origin of ChargeDensity Waves, Phys. Rev. Lett. 109, 086401 (2012).

[43] C. Putzke, J. Ayres, J. Buhot, S. Licciardello, N. E. Hussey, S. Friedemann, and A. Carrington, Charge Order and Superconductivity in Underdoped $\mathrm{YBa}_{2} \mathrm{Cu}_{3} \mathrm{O}_{7-\delta}$ under Pressure, Phys. Rev. Lett. 120, 117002 (2018).

[44] M. D. Watson, T. Yamashita, S. Kasahara, W. Knafo, M. Nardone, J. Béard, F. Hardy, A. McCollam, A. Narayanan, S. F. Blake et al., Dichotomy between the Hole and Electron Behavior in Multiband Superconductor FeSe Probed by Ultrahigh Magnetic Fields, Phys. Rev. Lett. 115, 027006 (2015).

[45] Data are available at the University of Bristol data repository, data.bris, at https://doi.org/10.5523/bris.1ga60qdfpvsi12ogxvaq14d6ht. 